\title{
PAPER
}

\section{Dystonia in multiple system atrophy}

\section{S M Boesch, G K Wenning, G Ransmayr, W Poewe}

J Neurol Neurosurg Psychiatry 2002;72:300-303

See end of article for authors' affiliations

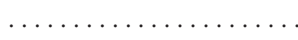

Correspondence to: Professor W Poewe, Department of Neurology, University Hospital, Anichstrasse 35, A-6020 Innsbruck, Austria; Werner.Poewe@uibk.ac.at

\section{Received}

28 November 2000

In revised form 25 May 2001

Accepted 26 June 2001
Objective: To delineate the frequency and nature of dystonia in multiple system atrophy (MSA). Methods: a cohort of 24 patients with clinically probable MSA over the past 10 years were prospectively followed up. Motor features were either dominated by parkinsonism (MSA-P subtype, $n=18$ ) or cerebellar ataxia (MSA-C, $n=6$ ). Classification of dystonic features and their changes with time was based on clinical observation during 6-12 monthly follow up visits. Parkinsonian features and complications of drug therapy were assessed. Most patients (22/24) died during the observation period. Neuropathological examination was confirmatory in all of the five necropsied patients.

Results: At first neurological visit dystonia was present in $11(46 \%)$ patients all of whom had been levodopa naive at this time point. Six patients (25\%) exhibited cervical dystonia (antecollis) (MSA-P $n=4, M S A-C n=2)$, five patients (21\%) showed unilateral limb dystonia (MSA-P $n=4 ; M S A-C n=1$ ). A definite initial response to levodopa treatment was seen in 15/18 patients with MSA-P, but in none of the six patients with MSA-C. A subgroup of 12 patients with MSA-P developed levodopa induced dyskinesias 2.3 years (range $0.5-4$ ) after initiation of levodopa therapy. Most patients had peak dose craniocervical dystonia; however, some patients experienced limb or generalised dystonia. Isolated peak dose limb chorea occurred in only one patient.

Conclusion: The prospective clinical study suggests that dystonia is common in untreated MSA-P. This finding may reflect younger age at disease onset and putaminal pathology in MSA-P. Levodopa induced dyskinesias were almost exclusively dystonic affecting predominantly craniocervical musculature. Future studies are required to elucidate the underlying pathophysiology of dystonia in MSA.
C linical features and natural history of multiple system atrophy (MSA) have been established in four recent series. ${ }^{1-4}$ Multiple system atrophy is usually defined by the predominance of parkinsonian (MSA-P type) or cerebellar (MSA-C type) features. ${ }^{56}$ According to a recent literature review dystonia is rare in postmortem confirmed MSA. ${ }^{7}$ We therefore prospectively investigated the frequency of dystonia and its relation to levodopa treatment in a group of 24 patients with MSA.

\section{PATIENTS AND METHODS \\ Clinical studies}

Twenty four consecutive patients with probable MSA according to the criteria of Quinn and Gilman were included in the study. ${ }^{56}$ Seventeen patients were women, and seven were men (ratio 2.4:1). Mean age at disease onset was 59 (SD 7.6) years (range 45-72 years) and mean disease duration defined as onset of symptoms to last follow up $(n=2)$ or death $(n=22)$ was 5.7 (SD 2.3) years (range 3-13 years). Eighteen out of 24 patients with MSA were classified as MSA-P type (75\%); the remaining six patients fulfilled the criteria of the MSA-C variant $(25 \%)$. Patients were repeatedly followed up at 6 to 12 monthly intervals for up to 10 years and the following clinical features were recorded:

(1) Presence of dystonia, its topographical distribution and clinical pattern (tonic versus phasic movements), and onset in relation to initiation of levodopa therapy (before levodopa $v$ after levodopa).

(2) Levodopa dose and motor response to levodopa using the following rating scale: nil to poor (improvement smaller than 30\%), moderate (improvement greater than 30\%), good (improvement greater than 50\%), or excellent (improvement greater than $70 \%$ ).

(3) Presence of non-dystonic levodopa induced dyskinesias, their topographical distribution, clinical pattern (choreic, myoclonic, or other) and time of onset in relation to initiation of levodopa.
Levodopa treatment was initiated in all patients with MSA and maintained in 15 of them throughout follow up. Neuropathological examination was confirmatory in the five necropsied patients.

\section{RESULTS}

\section{Dystonia before levodopa treatment}

Dystonia occurred in 11 (46\%) patients with MSA before levodopa exposure. All of the affected patients had focal dystonia. Antecollis was present in four of 18 patients with MSA-P and in two of six patients with MSA-C. Limb dystonia was present in four of 18 patients with MSA-P, and in one of six patients with MSA-C. It comprised writer's cramp or dystonic arm posturing in two patients each and equinovarus foot posturing in another patient. Laterality of limb dystonia and initial parkinsonian features corresponded in all patients. Antecollis was not modified by geste antagonistique manoeuvres in any patient.

\section{Response to levodopa treatment}

All patients $(n=24)$ received levodopa treatment. The average disease duration from symptom onset to the introduction of levodopa treatment was 1.5 (SD 0.5-3) years. If significant clinical benefit could not be obtained (improvement greater than $30 \%$ ) after several weeks on escalating dosages up to a maximum of $1200 \mathrm{mg} /$ day (if tolerated), drug treatment was slowly tapered. In nine (38\%) patients escalating levodopa dosages failed to elicit a motor response and therapy was therefore withdrawn. This group of non-responders to levodopa consisted of three patients with MSA-P and six patients with MSA-C. None of them developed levodopa induced dyskinesias.

Abbreviations: MSA, multiple system atrophy; MSA-P type, predominance of parkinsonian MSA; MSA-C type, cerebellar MSA 


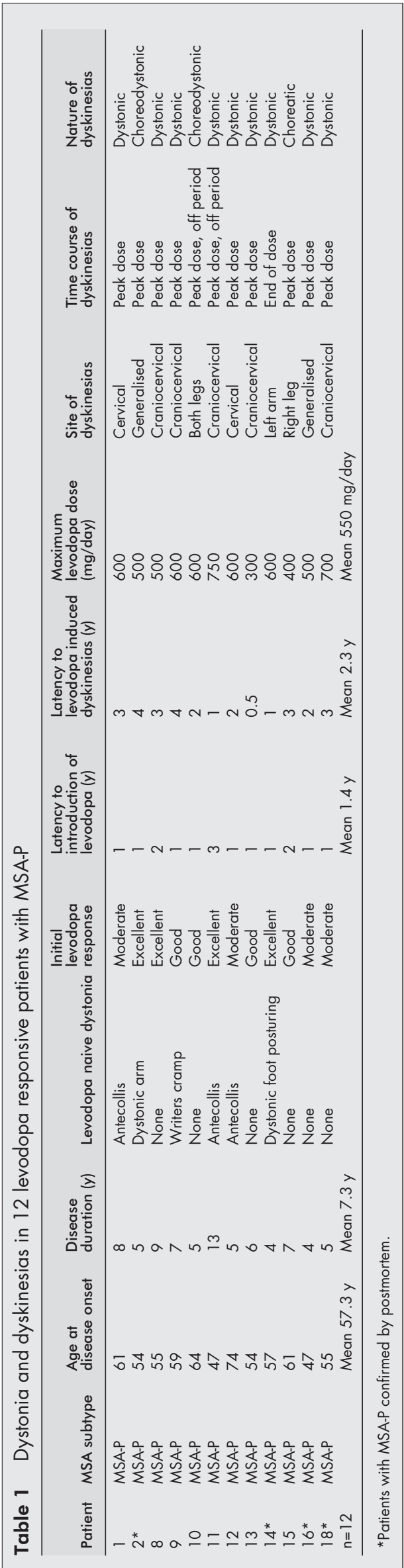

The levodopa responder group consisted of 15 (83\%) patients with MSA-P in whom initial response to levodopa therapy was scored as excellent in four $(27 \%)$, good in seven $(47 \%)$, or moderate in four patients $(27 \%)$ (table 1$)$. After 2 years of dopaminergic treatment only nine $(60 \%)$ patients were still considered responsive and this percentage declined to $13 \%$ (two patients) after 4 years. Levodopa induced motor fluctuations emerged in all 15 patients with MSA-P in the responder group. Unilateral limb dystonia was improved by levodopa in all patients presenting with this feature.

Three patients with MSA-P $(4,5,6)$ who showed a clear initial response to levodopa treatment never developed dyskinesias. They deteriorated rapidly due to severe autonomic failure and pyramidal involvement, and died 3-4 years after disease onset.

\section{Levodopa induced dystonia and dyskinesias}

A subgroup of $12(80 \%)$ patients with MSA-P developed dyskinesias on average 2.3 (SD 1.1) years after the initiation of levodopa therapy. The levodopa induced dyskinesias were predominantly $(n=2)$ or purely $(n=9)$ dystonic in all but one of these patients. Dyskinesias were interdose in all but one patient (14) who exhibited end of dose dystonia.

As shown in table 1, levodopa induced dyskinesias were confined to the neck and consisted of "on period" phasic dystonic posturing in seven of the 12 responsive patients with MSA-P. This group comprised all three patients with antecollis as presenting symptom in whom levodopa exposure induced phasic rotational head movements. Additional facial dystonia was present in five patients and consisted predominantly of spasms of the lower part of the face, lips, and eventually the platysma. In three patients levodopa induced dyskinesias were confined to the limbs, another two patients exhibited generalised dyskinesias.

\section{DISCUSSION}

Dystonia is typically encountered as a levodopa induced phenomenon in PD. ${ }^{8}$ Except from a literature review limited by poor documentation of clinical features dystonia has never been systematically studied in patients with MSA. ${ }^{7}$ Our study suggests that dystonia is more common in MSA than previously reported, occurring in $42 \%$ of levodopa naive patients. Although there are no prospective series assessing the frequency of dystonia in untreated PD, dystonia has been reported in $16 \%$ of levodopa naive patients with $\mathrm{PD}$ in a retrospective survey. ${ }^{8}$ This suggests that dystonia is more common in untreated MSA compared with untreated PD.

In keeping with previous reports dystonia in the present series of patients predominantly affected craniocervical muscles with antecollis being present in $25 \%$ of cases. ${ }^{6}{ }^{9-12}$ Importantly, unilateral limb dystonia was also seen, albeit in a minority of five $(21 \%)$ patients.

Disproportionate antecollis is considered a rare, but characteristic, "red flag" suggestive of MSA, and usually emerging late in the course of disease. ${ }^{67}$ In our study antecollis occurred in $25 \%$ of the patients and it often emerged within early disease stages. The discrepancy between the present finding and previous reports may well reflect differences in the clinical classification of abnormal head posturing. ${ }^{6}{ }^{13}$ We defined antecollis as abnormal neck flexion and may therefore have included patients with moderate posturing. However, there is no validated scale that would have allowed us to define a disproportionate degree of antecollis. In a previous neuropathological study of 10 patients with MSA antecollis was attributed to neuronal loss in the ventral putamen. ${ }^{14}$ Furthermore, studies on symptomatic dystonia emphasise the role of the putamen as a major lesion site in dystonia. ${ }^{15-19}$ Additional involvement of interconnections between the basal ganglia and the brain stem or cerebellar structures may contribute towards abnormal axial posturing and, thus, to dystonia in 
MSA. $^{20-24}$ Recently, marked myopathy of neck extensor muscles was found in patients with clinically probable MSA and antecollis. ${ }^{25}$ Further studies are required to determine the relevance of myopathic changes associated with neck muscle deformities in MSA.

Our study showed that limb dystonia may occur in levodopa naive MSA, similarly to previous reports in early PD. ${ }^{26-28}$ Because levodopa treatment abolished limb dystonia in all levodopa responsive patients with MSA it is likely that dystonia in levodopa naive de novo patients with MSA predominantly reflects early nigrostriatal dysfunction due to a decrease of endogenous dopaminergic stimulation rather than postsynaptic striatal pathology. ${ }^{29-31}$

We found a definite initial response to levodopa treatment in $83 \%$ of patients with MSA-P, which was scored excellent in $27 \%$. This finding is in line with data obtained by Wenning et al, who found a good or excellent response to levodopa treatment in $29 \%$ of patients with MSA-P. ${ }^{13}$ Levodopa induced dyskinesias developed in 12 out of 15 levodopa responsive patients with MSA-P after an average exposure of 2.3 years. The three patients with MSA-P without levodopa induced dyskinesias despite an initial antiparkinsonian response had rapidly progressive disease with death occurring within a few years, which may have precluded the evolution of motor complications resulting from chronic levodopa delivery. By contrast, recent prospective controlled trials of dopamine agonists versus levodopa in PD reported dyskinesia rates between $20 \%$ and $40 \%$ in the levodopa treatment arms over 3 to 5 years of treatment. ${ }^{32} 33$ Although the latency to onset of dyskinesias is comparable in the PD trials, in our present series patients with MSA-P seem to more prone to develop dyskinesias. Age at onset of disease and disease severity both determine the frequency and severity of dyskinesias in PD. ${ }^{34}$ Age at onset is about 1 decade earlier in MSA-P compared with $\mathrm{PD},{ }^{1}$ furthermore the motor disability at presentation is clearly more advanced in MSA-P and it progresses more rapidly than in PD. ${ }^{35}$ Both findings may partly account for the high rates of levodopa induced dyskinesias in MSA-P.

Our study confirms previous reports of the craniocervical predilection of levodopa induced dyskinesias. ${ }^{7101366}$ Furthermore, most of the dyskinesias found were dystonic. There is no clear explanation for the topographic predilection and dystonic nature of levodopa induced dyskinesias in MSA. Although the mechanism of levodopa induced dyskinesias is incompletely understood, an abnormal imbalance of activity in the indirect and direct putaminopallidal outflow systems, possibly related to levodopa induced changes in signal transaction in striatal medium spiny neurons, are thought to play an important part. ${ }^{37}$ In MSA-P degeneration in the caudal and dorsal part of the putamen, which receives topographical projections from cortical limb areas, may account for the lack of dyskinetic responses in the limbs when such patients are exposed to levodopa. In early disease stages; however, the ventral putaminal region, related to facial projections, is relatively spared providing the substrate for a dystonic face and neck response to levodopa. ${ }^{14}$

It is interesting to note that levodopa induced on period dyskinesias when they affect the orofacial region seem to be dystonic both in MSA and PD. ${ }^{8}$ This may be linked to the normal motor physiology of craniocervical muscles, which are predominantly used for slower phasic movements as in physiological grimacing, speaking, or chewing and have to perform less ballistic rapid movements compared with the limbs.

In summary, this prospective clinical study suggests that dystonia is often encountered in untreated MSA-P. The predominant occurrence of levodopa induced dystonia in the craniocervical region is probably related to the topography of putaminal pathology in this disorder.

\section{Authors' affiliations}

S M Boesch, G K Wenning, G Ransmayr, W Poewe, Department of Neurology, University Hospital, Innsbruck, Austria

\section{REFERENCES}

1 Wenning GK, Ben Shlomo Y, Magalhaes M, et al. Clinical features and natural history of multiple system atrophy: an analysis of 100 cases. Brain 1994;117:835-45.

2 Schulz JB, Klockgether T, Petersen D, et al. Multiple system atrophy: natural history, MRI morphology, and dopamine receptor imaging with ${ }^{23}$ IBZM-SPECT. J Neurol Neurosurg Psychiatry. 1994;57:1047-56.

3 Gouider-Khouja N, Vidailhet $M$, Bonnet $A M$, et al. "Pure" striatonigral degeneration and Parkinson's disease: a comparative clinical study. Mov Disord 1995; 10:288-94.

4 Albanese A, Colosimo C, Bentivoglio AR, et al. Multiple system atrophy presenting as parkinsonism: clinical features and diagnostic criteria. $J$ Neurol Neurosurg Psychiatry. 1995;59:144-51.

5 Gilman S, Low PA, Quinn NP, et al. Consensus statement on the diagnosis of multiple system atrophy. J Neurol Sci 1999;163:94-8.

6 Quinn N. Multiple system atrophy-the nature of the beast. J Neurol Neurosurg Psychiatry 1989;52(suppl):78-89.

7 Rivest J, Quinn N, Marsden CD. Dystonia in Parkinson's disease, multiple system atrophy, and progressive supranuclear palsy. Neurology 1990;40:1571-8.

8 Poewe WH, Lees AJ, Stern GM. Dystonia in Parkinson's disease: clinical and pharmacological features. Ann Neurol 1988;23:73-8.

9 Wenning GK, Ben-Shlomo Y, Hughes A, et al. What clinical features are most useful to distinguish definite multiple system atrophy from Parkinson's disease? J Neurol Neurosurg Psychiatry 2000;68:434-40.

10 Hughes AJ, Colosimo C, Kleedorfer B, et al. The dopaminergic response in multiple system atrophy. J Neurol Neurosurg Psychiatry 1992;55:1009-13

11 Gosset A, Pellissier JF, Delpuech F, et al. Striatonigral degeneration associated with olivopontocerebellar atrophy. Anatomo-clinical study of 3 cases. Nosologic discussion. Rev Neurol (Paris) 1983;139:125-39. (In French.)

12 Goetz CG, Tanner CM, Glantz R, et al. Bupropion in Parkinson's disease. Neurology 1984;34:1092-4.

13 Wenning GK, Tison F, Ben Shlomo Y, et al. Multiple system atrophy: a review of 203 pathologically proven cases. Mov Disord 1997; 12:133-47

14 Fearnley JM, Lees AJ. Striatonigral degeneration: a clinicopathological study. Brain 1990;113:1823-42.

15 Bhatia KP, Marsden CD. The behavioural and motor consequences of focal lesions of the basal ganglia in man. Brain 1994;117:859-76.

16 Lehericy S, Vidailhet M, Dormont D, et al. Striatopallidal and thalamic dystonia. A magnetic resonance imaging anatomoclinical study. Arch Neurol 1996:53:241-50.

17 Krauss JK, Pohle T, Weber S, et al. Bilateral stimulation of globus pallidus internus for treatment of cervical dystonia. Lancet 1999;354:837-8

18 Krystkowiak P, Martinant P, Defebre L, et al. Dystonia after striatopallidal and thalamic stroke: clinicoradiological correlations and pathophysiological mechanisms. J Neurol Neurosurg Psychiatry 1998;65:703-8

19 Perlmutter JS, Stambuck MK, Markham J, et al. Decreased $\left[{ }^{18} \mathrm{~F}\right]$ spiperone binding in putamen in idiopathic focal dystonia. $J$ Neurosci 1997: 17:843-50.

20 Berardelli A, Rothwell JC, Hallett M, et al. The pathophysiology of primary dystonia. Brain 1998;121:1195-212.

21 Hallett $M$. The neurophysiology of dystonia. Arch Neurol 1998;55:601-3.

22 Eidelberg D. Functional brain networks in movement disorders. Curr Opin Neurol 1998;11:319-26.

23 LeDoux MS, Hurst DC, Lorden JF. Single-unit activity of cerebellar nuclear cells in the awake genetically dystonic rat. Neuroscience 1998; 86:533-45.

24 Odergren T, Stone-Elander S, Ingvar M. Cerebral and cerebellar activation in correlation to the action-induced dystonia in writer's cramp. Mov Disord 1998; 13:497-508.

25 Askmark $\mathbf{H}$, Eeg-olofsson $\mathrm{KE}$, Johansson $\mathrm{A}$, et al. Parkinsonism and neck extensor myopathy. A new syndrome or coincidental findings? Arch Neurol 2001;58:232-7.

26 Purves Stewart J. Paralysis agitans; with an account of a new symptom. Lancet 1898; ii: 1258-60.

27 Gowers WR. A manual of diseases of the nervous system. London, Churchill, 1888:589-607.

28 Poewe W, Lees AJ, Steiger D, et al. Foot dystonia in Parkinson's disease: clinical phenomenology and neuropharmacology. Adv Neurol 1987;45:357-60

29 Poewe WH, Wenning GK. The natural history of Parkinson's disease. Ann Neurol 1998;44(suppl):S1-9.

30 Schober R, Langston JW, Forno LS. Idiopathic orthostatic hypotension: biochemical and pathologic observations in 2 cases. Eur Neurol 1975;13:177-88

31 Boudin G, Guillard A, Mikol J, et al. Striato-nigral degeneration: apropos of a clinical, therapeutic, and anatomic study of 2 cases. Rev Neurol (Paris) 1976;132:137-56. 
32 Rascol O Brooks DJ, Korzyn AD, et al. A 5 year study of the incidence of dyskinesia in patients with early Parkinson's disease who were treated with ropinirole or levodopa. 056 Study Group. N Engl J Med 2000;342:1484-91.

33 Ruotinnen HM, Rinne UK. COMT Inhibition in the treatment of Parkinson's disease. J Neurol 1998;245(suppl 3):25-34.

34 Schrag A, Ben-Shlomo Y, Brown R, et al. Young-onset Parkinson's disease revisted: clinical features, natural history, and mortality. Mov Disord 1998;13:885-94.
35 Mueller J, Wenning GK, Jellinger K, et al. Progression of Hoehn and Yahr stages in parkinsonian disorders: a clinicopathologic study. Neurology 2000;55:888-9

36 Testa D, Filippini G, Farinotti $M$, et al. Survival in multiple system atrophy: a study of prognostic factors in 59 cases. J Neurol 1996:243:401-4

37 Henry B, Fox SH, Peggs D, et al. The $\alpha 2$-adrenergic receptor antagonist idazoxan reduces dyskinesia and enhances anti-parkinsonian actions of L-dopa in the MPTP-lesioned primate model of Parkinson's disease. Mov Disord 1999;14:744-53.

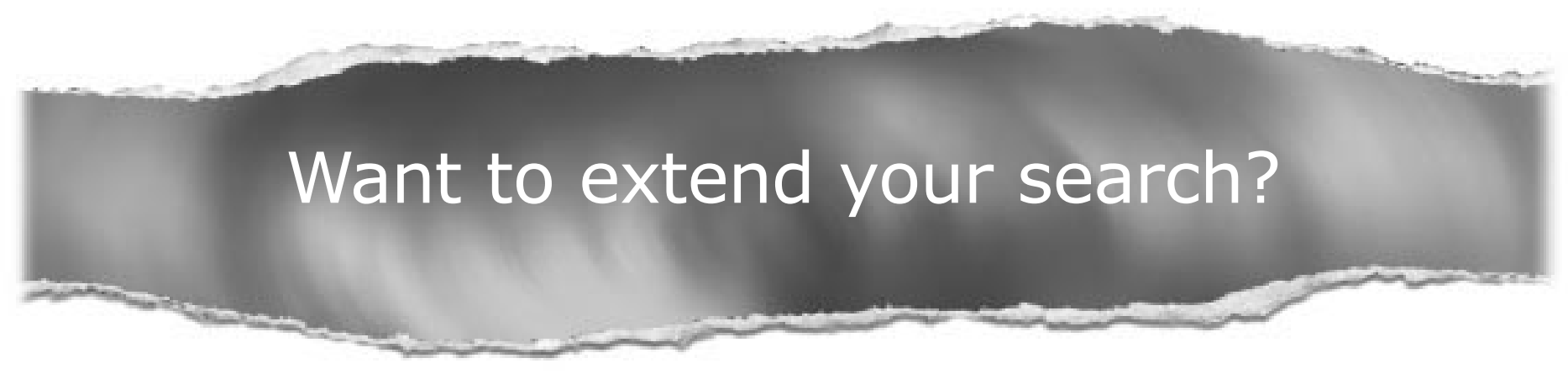

Cross journal searching

If you can't find what you are looking for in the Journal of Neurology, Neurosurgery, and Psychiatry you can extend your search across many of the more than 200 journals available for selection. You can restrict your search to specific subject areas (eg, clinical medicine, basic research), or select specific journals, or search all available titles.

\section{www.jnnp.com}

